\title{
Development of a contactless DC current sensor with high linearity and sensitivity based on the magnetoelectric effect
}

\author{
N. Castro ${ }^{a, b}$, S. Reis ${ }^{\text {a,b }}$, M.P. Silva ${ }^{a, b}$, V. Correia ${ }^{a, b}$, , S. Lanceros-Mendez ${ }^{\text {a,c,d, , }}$ \\ P.Martins ${ }^{\mathrm{a}}$ \\ ${ }^{a}$ Centro/Departamento de Física, Universidade do Minho, 4710-057 Braga, Portugal \\ ${ }^{\mathrm{b}}$ Centro Algoritmi, Universidade do Minho, 4800-058 Guimarães, Portugal \\ ${ }^{\mathrm{c}}$ BCMaterials, Parque Científico y Tecnológico de Bizkaia, 48160, Derio, Spain \\ dIKERBASQUE, Basque Foundation for Science, 48013 Bilbao, Spain
}

\section{Abstract}

The magnetoelectric (ME) effect is increasingly being considered an attractive alternative for magnetic field and current sensing, being able to sense static and dynamic magnetic fields.

The present work reports on a DC current sensor device based on a ME PVDF/Metglas composite, a solenoid and the corresponding electronic instrumentation.

The ME sample shows a maximum ME coefficient $\left(\alpha_{33}\right)$ of $34.48 \mathrm{~V} \cdot \mathrm{cm}^{-1} \cdot \mathrm{Oe}^{-1}$, a linear response $\left(\mathrm{R}^{2}=0.998\right)$ and a sensitivity of $6.7 \mathrm{mV} . \mathrm{A}^{-1}$. With the incorporation of a charge amplifier, an AC-RMS converter and a microcontroller the linearity is maintained $\left(\mathrm{R}^{2}=0.997\right)$, the ME output voltage increases to a maximum of $2320 \mathrm{mV}$ and the sensitivity rises to $476.5 \mathrm{mV} . \mathrm{A}^{-1}$. Such features allied to the highest sensitivity reported in the literature on polymer-based magnetoelectric composites provides the reported ME sensing device suitable characteristics to be used in non-contact electric current measurement, motor operational status checking, and condition monitoring of rechargeable batteries, among others.

Keywords:

\section{Introduction}

Current sensors are widely used in houses, cars and industrial applications. They are essential for protection and control of motors, pumps and overcurrent protectors. 
Furthermore, they take an important role in production, conversion and energy storage $[1,2]$.

Current sensors can be classified based upon the underlying fundamental physical principle: Ohm's law of resistance sensors, Faraday's law of induction sensors, magnetic field sensors or Faraday effect sensors [3]. The most commonly used current sensor is the shunt resistor, which is based on Ohm's law. Its simplicity and ability to measure both $\mathrm{AC}$ and DC currents are the main advantages of this kind of sensor, however it must be introduced into the current conducting wire, which may generate substantial power loss. Other widely used current sensor is the current transformer, based on Faraday's law of induction. This sensor provides electrical isolation between the measured current and the output signal, but it is unable of measuring DC currents [3]. Moreover in the traditional approaches for current sensing such as ring-type [4] or close magnetic circuit type [5] sensors, the conducting wire should pass through the centre of the ring or the sensor needs to be inserted in series, forcing the wire to be disconnected. These facts lead to complicated measurement procedures.

Magnetic field sensors represent an increasingly attractive alternative for current sensing, being able to sense static and dynamic magnetic fields and ensure the isolation from the current being measured [1, 3]. Hall effect sensors are the most popular and widely used magnetic current sensor mainly due to their compatibility with microelectronic devices [6]. Nonetheless, it has relevant drawbacks such as an offset voltage, which may vary with supply voltage, temperature and stress. In this way, additional circuitry for offset reduction, temperature compensation and signal amplification are required, being the power consumption of these sensors between 100 and $200 \mathrm{~mW}$ [6-8]. Anisotropic magnetoresistance (AMR) and giant magnetoresistance (GMR) sensors are also used as magnetic current sensors, where the electrical resistance varies as a function of the magnetic field. They are much more sensitive than Hall effect sensor, in particular the GMR sensors, but need to be configured within a Wheatstone bridge configuration [3]. The main problems of AMR sensors are related to high thermal drift and non-linearity. GMR sensors also exhibit a non-linear behaviour, high dependence with temperature and no stable hysteresis. However, for GMR sensor there is no "geometric trick" to reduce the non-linearity, such as for AMR sensors, which can be arranged in a barber-pole configuration to overcome this problem. Furthermore a very strong magnetic field can permanently modify the sensor behaviour and damage the GMR sensor $[1,3,9,10]$. 
With the potential to solve the abovementioned sensing problems, magnetoelectric (ME) composites have drawn increasing interest in the development of innovative current sensors [11, 12].

Among the different ME composite structures, laminated composites, comprising bonded piezoelectric and magnetostrictive layers, are the ones with the highest ME response, thus being the most suitable materials for sensor development [13]. In such ME laminates, the ME effect results from the cross interaction between the piezoelectric and magnetostrictive phases. It is an extrinsic effect, which depends on the composite microstructure and occur via the elastic coupling of the phases [14, 15]. ME current sensors are based on the Ampère's law: when an electric current is applied to an electrical conductor, a magnetic field (Equation 1) is induced given by [16].

$$
H=\frac{\mu I}{2 \pi R}
$$

As these current sensors are based on magnetic sensing, they possess the advantages such as electrical isolation and the ability to measure AC and DC currents [15, 17]. Some pioneering work in ME current sensors have been carried out based on ME composites $[4,17]$, however, until now, all those reports use a ceramic piezoelectric phase, which is rigid, fragile and shows high dielectric losses, hindering applicability [11, 14]. Actual industry demands for flexible, non-brittle and cheap materials with low temperature fabrication process, can be met by polymer-based ME composites [14].

In this way, this work reports on the development of a current sensor fabricated from a polymer-based ME laminate based on Metglas and poly(vinylidenefluoride) (PVDF), combined with an electronic circuit to process the acquired signal. The latter is composed by a charge amplifier, an AC-RMS converter and a microcontroller. The selection of the Metglas/PVDF composite is related to the fact that this combination provide the highest ME response and magnetic sensitivity among polymer-based ME materials [14]. Furthermore, Metglas shows high sensitivity, low noise, high magnetic permeability and high piezomagnetic coefficient [18] and PVDF shows the highest piezoelectric coefficient among polymers, chemical stability, flexibility, large electrical resistivity and low dielectric losses [19, 20]. As the signal generated by the ME composite is in the form of a charge variation, and susceptible to noise, a charge amplifier was used to decrease such noise and convert the signal into a voltage signal, amplifying the initial amplitude and filtering lower frequency noise [21, 22].

\section{Experimental}




\subsection{Magnetoelectric composite fabrication}

The polymer-based ME laminate was produced by bonding both PVDF and Metglas layers with M-Bond 600 epoxy (Vishay Precision Group) under vacuum during 3 hours at $50^{\circ} \mathrm{C}$. The ME laminate structure was composed by a piezoelectric layer of a commercial $\beta$-PVDF (Measurement Specialties, USA) with dimensions of $10 \mathrm{~mm}$ x 30 $\mathrm{mm} \times 52 \mu \mathrm{m}$, poled along the thickness direction, and a magnetostrictive layer of Metglas (2605SA1, Hitachi Metals Europe GmbH, Germany) with dimensions of $5 \mathrm{~mm}$ x $27 \mathrm{~mm}$ x $25 \mu \mathrm{m}$, magnetized along the longitudinal direction.

\subsection{Characterization}

Magnetoelectric response characterization was performed in a system composed by two Helmholtz coils in order to generate a $\mathrm{H}_{\mathrm{DC}}$ ranging from 0 Oe to $22 \mathrm{Oe}$, via a DC input current (Keithley 2400), and a superimposed $\mathrm{H}_{\mathrm{AC}}$ of 0.3 Oe produced via an AC current (Agilent 33220A signal generator).

Samples were characterized by measuring the voltage induced in the piezoelectric layer by the magnetic field variation. Since the ME laminates are susceptible to electromagnetic noise, a lock-in amplifier (Stanford Research SR530) was used to filter the ME signal through a narrow band pass filter.

To find the electromechanical resonance frequency of the sample, $\mathrm{H}_{\mathrm{DC}}$ and $\mathrm{H}_{\mathrm{AC}}$ values were kept constant ( $3.45 \mathrm{Oe}$ and $0.3 \mathrm{Oe}$, respectively) and the frequency was varied from $1 \mathrm{kHz}$ to $60 \mathrm{kHz}$. The optimum $\mathrm{H}_{\mathrm{DC}}$ was achieved by maintaining the $\mathrm{H}_{\mathrm{AC}}$ and frequency values constant ( 0.3 Oe and $28.4 \mathrm{kHz}$, respectively). The ME coefficient $\left(\alpha_{33}\right)$ was calculated based on equation 2:

$$
\alpha_{33}=\frac{\Delta V}{t \cdot H_{A C}}
$$

where $\Delta \mathrm{V}, \mathrm{t}$ and $\mathrm{H}_{\mathrm{AC}}$ are the induced ME voltage, the PVDF thickness and the applied AC magnetic field value, respectively.

The device current sensing experiments were performed in a system composed by the ME laminate, a sample holder with a solenoid in order to generate a $\mathrm{H}_{\mathrm{AC}}$ magnetic field of $1 \mathrm{Oe}$, and the electronic readout system (Figure 1a). Aiming simulate real conditions, a copper wire was used in which an IDC current was applied ranging from $0 \mathrm{~A}$ to $5 \mathrm{~A}$ (Figure $1 \mathrm{~b}$ ), the induced field being calculated by Equation 1. The system also allows the control of the distance between current sensor and the current wire. 


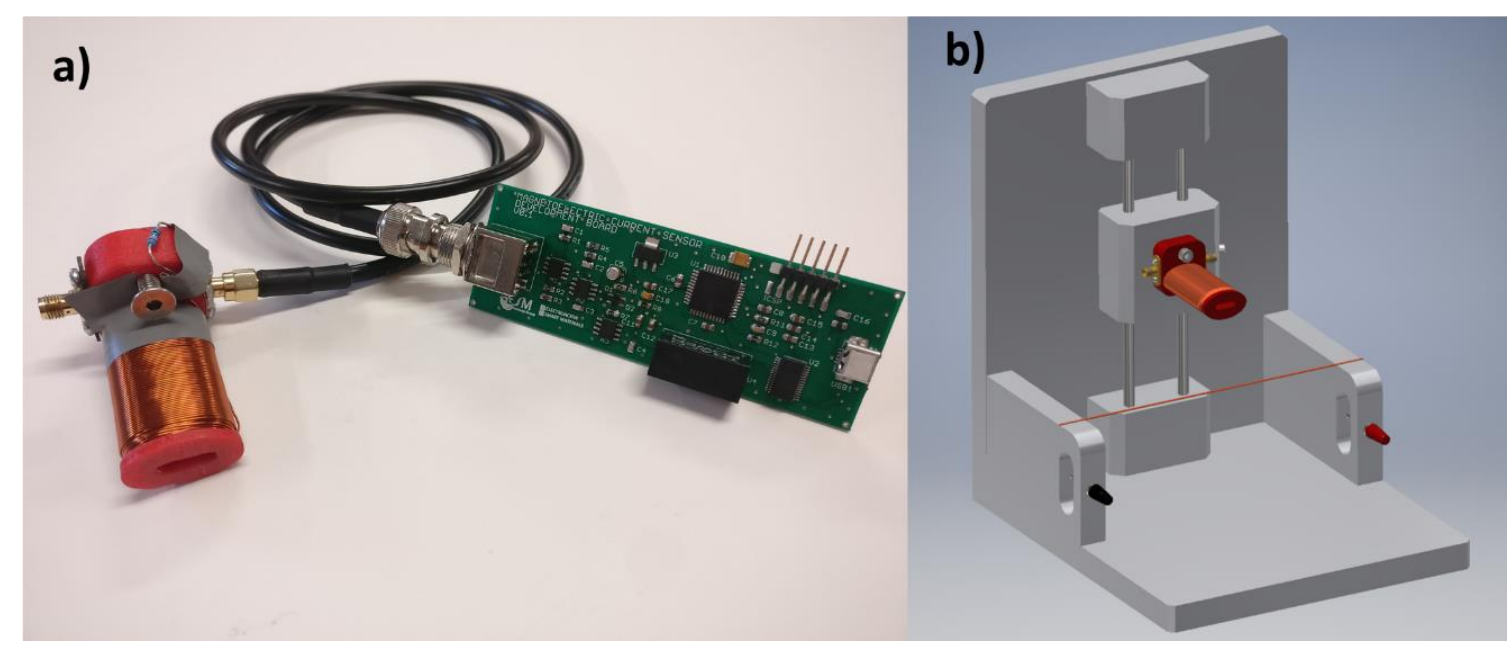

Figure 1. a) Left: ME laminate inside a sample holder with a solenoid; Right: Electronic components of the sensing device. b) Sensor testing bench composed by a copper wire used to apply the testing current and a moving system allowing the precise control of distance between current sensor and the copper wire.

The purpose of the sensor is to measure the IDC current in the conductor wire. Having a charge response signal from the piezoelectric layer, the implemented electronic instrumentation solution has two conversion stages, a charge amplifier for a charge/voltage conversion and signal amplification, based on the readout circuit presented in [23]. For signal output optimization and low digital analysis requirements it was used an AC/DC conversion circuit that outputs the amplified ME signal (AC $\mathrm{RMS}$ value).

After the analogue circuit, a microcontroller was used to read the sensor data through its analogue-to-digital converter and send the sensor data to a computational terminal, where the data is treated and properly presented.

\section{Results and Discussion}

The ME composite sample was characterized in terms of resonant frequency, signal amplitude and magnetic field range. Figure 2 illustrates the ME sample response to $\mathrm{H}_{\mathrm{DC}}$ and $\mathrm{H}_{\mathrm{AC}}$ variations. 

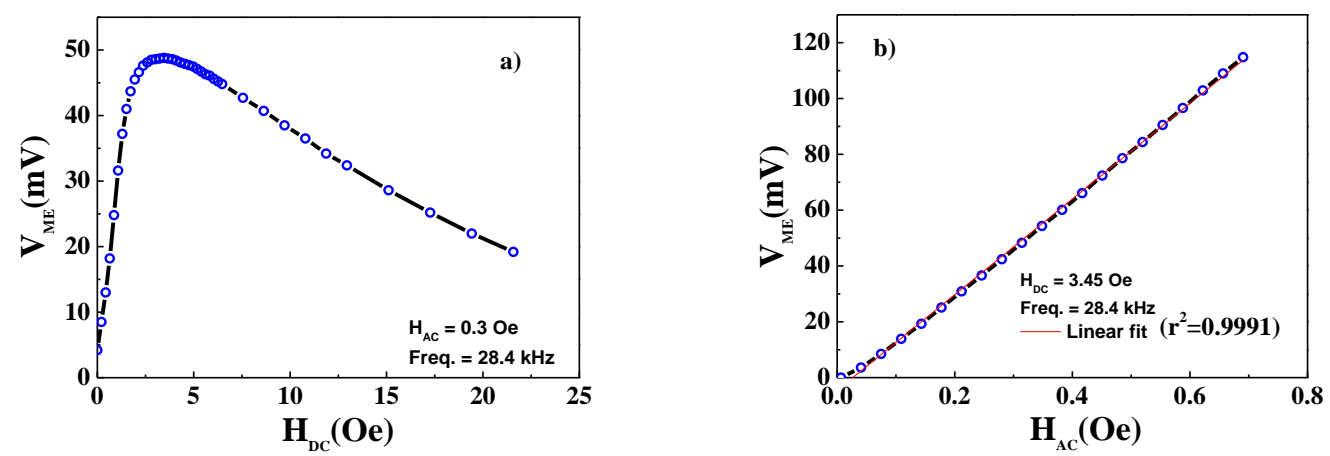

Figure 2. a) ME sample output response as a function of the $\mathrm{H}_{\mathrm{DC}}$, setting the $\mathrm{H}_{\mathrm{AC}}$ field to $0.3 \mathrm{Oe}$ at resonance frequency of $28.4 \mathrm{kHz}$; b) $\mathrm{ME}$ sample output response as a function of the $\mathrm{H}_{\mathrm{AC}}$, setting the $\mathrm{H}_{\mathrm{DC}}$ field to $3.45 \mathrm{Oe}$ and a frequency of $28.4 \mathrm{kHz}$.

Figure 2a shows that the ME voltage increases with increasing applied $\mathrm{H}_{\mathrm{DC}}$ until 3.45 Oe when a maximum ME voltage of $48.8 \mathrm{mV}$ is reached. A maximum $\mathrm{ME}$ coefficient ( $\alpha_{33}$ - determined from Eq. 1) of $31.28 \mathrm{~V} \cdot \mathrm{cm}^{-1} \cdot \mathrm{Oe}^{-1}$ is obtained for such DC magnetic field. This behavior is related with the increase of the piezomagnetic coefficient until the optimum DC magnetic field is reached. With further increase of the DC magnetic field, a decrease of the induced voltage is observed, resulting from the saturation of the magnetostrictive response. Figure $2 \mathrm{~b}$ shows that the ME voltage increases linearly with increasing applied $\mathrm{H}_{\mathrm{AC}}$ magnetic until 0.7 Oe when a maximum ME voltage of $114.8 \mathrm{mV}$ is reached. This linearity $\left(r^{2}=0.9991\right)$ is a suitable advantage for the use of these composites for sensor devices.

The response of the ME sensors as a function of the IDC was also evaluated (Figure 3 ), in order to validate the ME current sensor based on the ME effect.
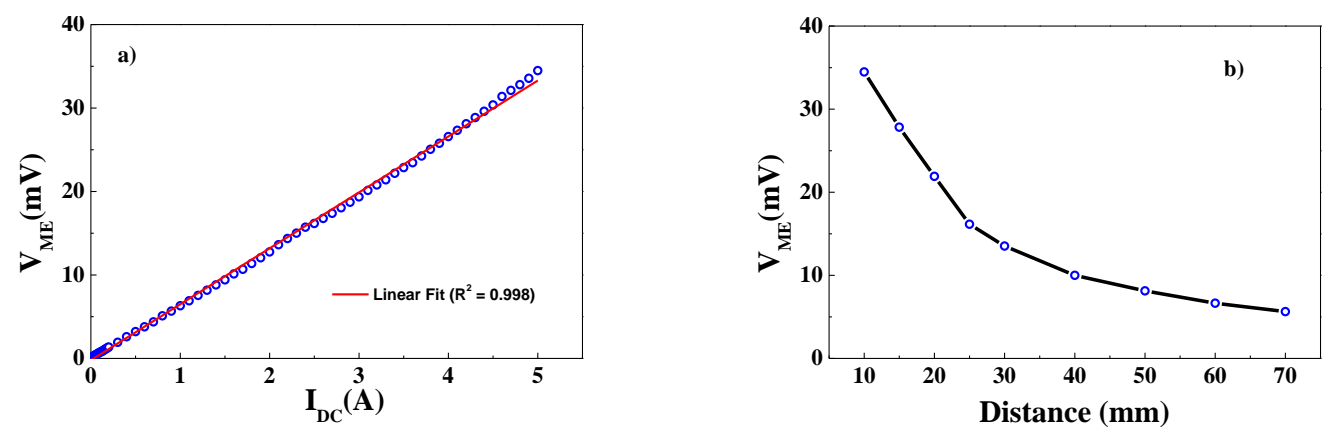

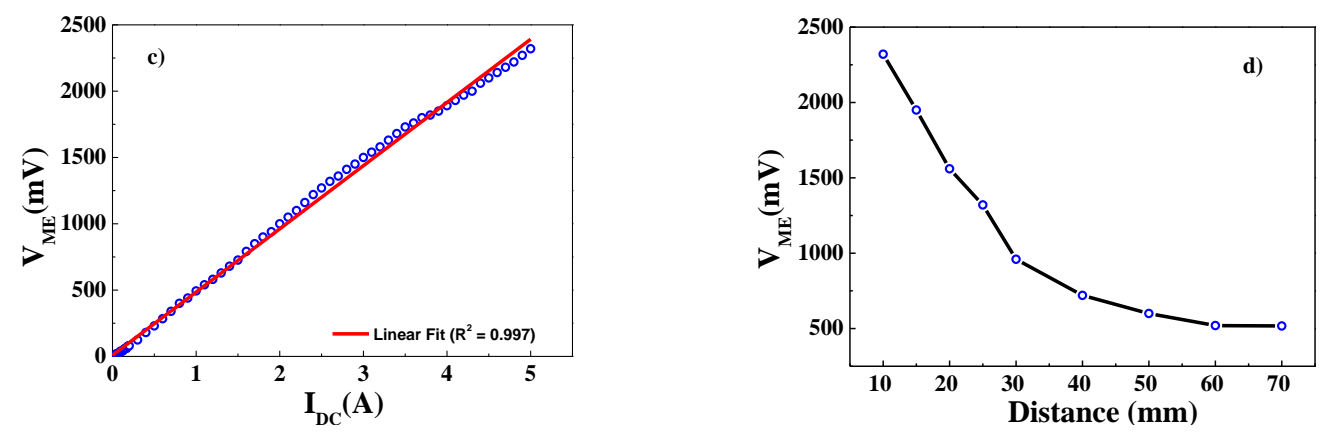

Figure 3. a) ME voltage response of the composite sample in the range $0<\mathrm{IDC}_{\mathrm{DC}}<5 \mathrm{~A}$, applying and $\mathrm{H}_{\mathrm{AC}}$ field of $1 \mathrm{Oe}$ at $26.1 \mathrm{kHz}$; b) ME voltage response of the composite as a function of the distance between the wire and the sensor device (from $10 \mathrm{~mm}$ to $70 \mathrm{~mm}$ ); c) ME voltage response of the final device in the range $0<\mathrm{I}_{\mathrm{DC}}<5 \mathrm{~A}$, applying and $\mathrm{H}_{\mathrm{AC}}$ field of $1 \mathrm{Oe}$ at $26.1 \mathrm{kHz}$; d) $\mathrm{ME}$ voltage response of the final device as a function of the distance between the wire and the sensor device (from $10 \mathrm{~mm}$ to 70 $\mathrm{mm})$.

Figure 3a demonstrates that the ME response of the composite sample increases with increasing $\mathrm{I}_{\mathrm{DC}}$ current up to $5 \mathrm{~A}$, at which the maximum ME voltage is reached $(34.48 \mathrm{mV})$. The sample exhibits a sensitivity of $6.7 \mathrm{mV} . \mathrm{A}^{-1}$ and a linear behavior between $0 \mathrm{~A}$ and $5 \mathrm{~A}\left(\mathrm{R}^{2}=0.998\right)$. Figure $3 \mathrm{~b}$ reveals that the ME voltage decreases with the distance from the wire ( $34.48 \mathrm{mV}$ to $5.63 \mathrm{mV}$ ).

Figure $3 \mathrm{c}$ and figure $3 \mathrm{~d}$ reveals an increase of the ME voltage response to a maximum of $2320 \mathrm{mV}$ after the electronic amplification and the signal conditioning circuit has been implemnted. After such electronic amplification, the linearity is maintained $\left(\mathrm{R}^{2}=0.997\right)$, and the sensitivity increased two orders of magnitude (476.5 $\left.m V . A^{-1}\right)$. In this way, the implementation of an amplifier with an optimized power supply rejection ratio does not influences the good linearity and increases the sensitivity of the original signal produced by the ME composite sample. Thereby it is concluded that, for this current range, the developed ME sensor has a large potential for actual current sensing due to its linearity and sensitivity.

\section{Conclusions}

The development of a DC current sensor composed by a Metglas/PVDF ME composite as an active sensing element is reported. An electronic instrumentation solution was also employed through the use of two conversion stages, charge/voltage conversion with signal amplification and an analogue-to-digital converter.

It was found that the maximum $\mathrm{V}_{\mathrm{ME}}$ occurs at $3,45 \mathrm{H}_{\mathrm{DC}}$ and a maximum $\alpha_{33}$ of $31.28 \mathrm{~V} . \mathrm{cm}^{-1} . \mathrm{Oe}^{-1}$ has been found for the such $\mathrm{H}_{\mathrm{DC}}$. 
After the incorporation of the electronic amplification circuit, the linearity is maintained $\left(\mathrm{R}^{2}=0.997\right)$ and the sensitivity increased from $6.7 \mathrm{mV} \cdot \mathrm{A}^{-1}$ to $476.5 \mathrm{mV} . \mathrm{A}^{-1}$.It is also found that the $\mathrm{V}_{\mathrm{ME}}$ response decreases with increasing distance from $10 \mathrm{~mm}$ to 70 $\mathrm{mm}$ : from $34.48 \mathrm{mV}$ to $5.63 \mathrm{mV}$ and from $2320 \mathrm{mV}$ to $517 \mathrm{mV}$ for the sensor without electronic instrumentation and with electronic instrumentation, respectively.

It is concluded that, for this current range, the fabricated ME sensor device shows suitable characteristics to be applied in current sensing applications, in particular in multifunctional flexible devices once it has a good output linearity and the highest sensitivity reported in the literature for polymer-based composites.

\section{ACKNOWLEDGMENTS}

The authors thank the FCT- Fundação para a Ciência e Tecnologia- for financial support in the framework of the Strategic Funding UID/FIS/04650/2013 and under project PTDC/EEI-SII/5582/2014. P.M., V.C., S.R. and M.S. acknowledges also support from FCT (SFRH/BPD/96227/2013, SFRH/BPD/97739/2013, SFRH/BDE/406 and SFRH/BD/70303/2010 grants respectively). Financial support from the Basque Government Industry Department under the ELKARTEK Program is also acknowledged. 


\section{References}

1. Ripka, P., Electric current sensors: A review. Measurement Science and Technology, 2010. 21(11).

2. Xiao, C., et al. An Overview of Integratable Current Sensor Technologies. in Conference Record - IAS Annual Meeting (IEEE Industry Applications Society). 2003.

3. Ziegler, S., et al., Current sensing techniques: A review. IEEE Sensors Journal, 2009. 9(4): p. 354-376.

4. $\quad$ Dong, S., J.F. Li, and D. Viehland, Vortex magnetic field sensor based on ringtype magnetoelectric laminate. Applied Physics Letters, 2004. 85(12): p. 23072309.

5. Zhang, J., et al., High-resolution current sensor utilizing nanocrystalline alloy and magnetoelectric laminate composite. Review of Scientific Instruments, 2012. 83(11).

6. Popovic, R.S., Z. Randjelovic, and D. Manic, Integrated Hall-effect magnetic sensors. Sensors and Actuators, A: Physical, 2001. 91(1-2): p. 46-50.

7. Herrera-May, A.L., et al., Resonant magnetic field sensors based on MEMS technology. Sensors, 2009. 9(10): p. 7785-7813.

8. Reis, S., et al., Optimized anisotropic magnetoelectric response of

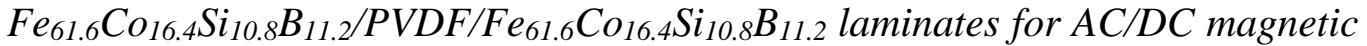
field sensing. Smart Materials and Structures, 2016. 25(5).

9. Bernieri, A., et al., Improving performance of gmr sensors. IEEE Sensors Journal, 2013. 13(11): p. 4513-4521.

10. Ripka, P. and M. Janošek, Advances in magnetic field sensors. IEEE Sensors Journal, 2010. 10(6): p. 1108-1116.

11. Le, M.Q., et al., Enhanced magnetoelectric effect for flexible current sensor applications. Journal of Applied Physics, 2014. 115(19).

12. Petrov, R.V., et al. Current sensor based on magnetoelectric effect. in 2014 18th International Symposium on Electrical Apparatus and Technologies, SIELA 2014 - Proceedings. 2014.

13. Reis, S., et al., Characterization of Metglas/poly(vinylidene fluoride)/Metglas magnetoelectric laminates for AC/DC magnetic sensor applications. Materials and Design, 2016. 92: p. 906-910.

14. Martins, P. and S. Lanceros-Méndez, Polymer-based magnetoelectric materials. Advanced Functional Materials, 2013. 23(27): p. 3371-3385.

15. Nan, C.W., et al., Multiferroic magnetoelectric composites: Historical perspective, status, and future directions. Journal of Applied Physics, 2008. 103(3).

16. Zhang, J.T., Y.M. Wen, and P. Li, A passive current sensor employing self-biased magnetoelectric transducer and high-permeability nanocrystalline flux concentrator. Rare Metals, 2015.

17. Dong, S., et al., Circumferential-mode, quasi-ring-type, magnetoelectric laminate composite-a highly sensitive electric current andor vortex magnetic field sensor. Applied Physics Letters, 2005. 86(18): p. 1-3.

18. Zhai, J., et al., Giant magnetoelectric effect in Metglas/polyvinylidene-fluoride laminates. Applied Physics Letters, 2006. 89(8).

19. Martins, P., A.C. Lopes, and S. Lanceros-Mendez, Electroactive phases of poly(vinylidene fluoride): Determination, processing and applications. Progress in Polymer Science, 2014. 39(4): p. 683-706.

20. Silva, M.P., et al., Stability of the electroactive response of $\beta$-poly(vinylidene fluoride) for applications in the petrochemical industry. Polymer Testing, 2010. 29(5): p. 613-615. 
21. Xing, Z.P., et al., Modeling and detection of quasi-static nanotesla magnetic field variations using magnetoelectric laminate sensors. Measurement Science and Technology, 2008. 19(1).

22. Zhuang, X., et al., Tunable Magnetoelectric Bending Resonance for Sensing Static Magnetic Fields. IEEE Sensors Journal, 2016. 16(3): p. 662-669.

23. Reis, S., et al., Fabrication and characterization of high-performance polymerbased magnetoelectric DC Magnetic Field sensors devices. IEEE Transactions on Industrial Electronics, 2017. PP(99): p. 1-1. 